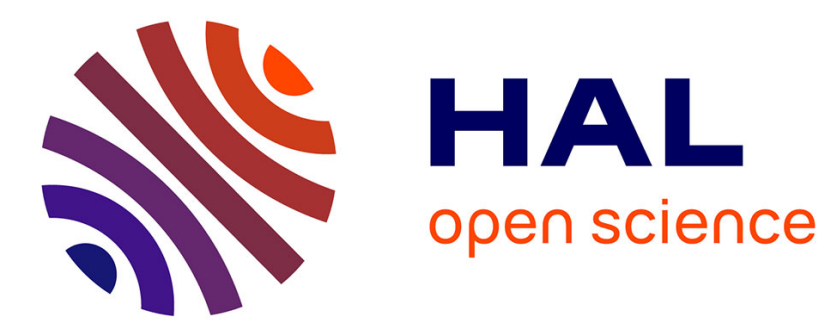

\title{
Une justice d'État trop sensible aux bruits de la rue (1947-1953)
}

\author{
Alain Bancaud
}

\section{To cite this version:}

Alain Bancaud. Une justice d'État trop sensible aux bruits de la rue (1947-1953). Sous la direction de Philippe Buton, Olivier Büttner, Michel Hastings. La Guerre froide vue d'en bas, CNRS Editions, pp.55-71, 2014, 9782271078230. halshs-01257821

\section{HAL Id: halshs-01257821 https://shs.hal.science/halshs-01257821}

Submitted on 18 Jan 2016

HAL is a multi-disciplinary open access archive for the deposit and dissemination of scientific research documents, whether they are published or not. The documents may come from teaching and research institutions in France or abroad, or from public or private research centers.
L'archive ouverte pluridisciplinaire HAL, est destinée au dépôt et à la diffusion de documents scientifiques de niveau recherche, publiés ou non, émanant des établissements d'enseignement et de recherche français ou étrangers, des laboratoires publics ou privés. 


\section{Une justice d'État trop sensible aux bruits de la rue (1947-1953)}

Alain Bancaud

Conformément à une tradition de gestion des crises politiques et sociales, le traitement juridique et judiciaire de la période " chaude » de la Guerre froide (1947-1953) échappe au «droit commun ». Sans pour autant correspondre au dispositif des temps les plus exceptionnels, tels les guerres ou les changements de régime, marqués par la création de législations et de juridictions extraordinaires de circonstance, comme on le voit sous la Révolution, la Restauration, Napoléon III, la Première Guerre mondiale, Vichy, la Libération, la guerre d'Algérie. Il s'agit en quelque sorte d'un dispositif ordinaire d'exception qui banalise la Guerre froide. Cette normalisation est renforcée par le travail des magistrats civils qui interviennent avec les juges militaires. Ils n'appréhendent plus le communisme comme ils ont pu le faire, un temps, dans les années $1920^{1}$ et sous Vichy ${ }^{2}$ : ils ramènent les affaires à des questions classiques, à la fois dépolitisées, individualisées, susceptibles de circonstances atténuantes, d'ordre public interne ou de nature politique et syndicale. Cette tendance est particulièrement sensible chez les magistrats d' « en bas ». Elle se retrouve au cours de l'histoire, même sous Vichy, et explique pourquoi la critique adressée par les ministres et les préfets aux magistrats et à leur manque de sens de la défense de l'État est finalement récurrente.

1. Monier Frédéric, Le complot dans la République, Paris, La Découverte, 1998. Dans la continuité de ce travail, voir la thèse qui nous a été très utile de Girard Pascal Les complots politiques en France et en Italie de la fin de la Seconde Guerre mondiale à la fin des années 1950, Thèse, Florence, Institut universitaire européen, 2012. 2. Bancaud Alain, Une exception ordinaire, Paris, Gallimard, « Essais », 2002. 


\section{UN DISPOSITIF JUDICIAIRE ET JURIDIQUE ORDINAIRE D’EXCEPTION}

À la différence d'autres événements, la Guerre froide n’entraine la création d'aucune juridiction d'exception ad hoc. Même la Haute Cour de justice de la IV République, juridiction politique dérogatoire installée à titre permanent par la Constitution de 1946, est exclue en raison de sa compétence limitée à la seule responsabilité des ministres et du chef de l'État alors que celle de la III République, jugeant aussi les atteintes à la sûreté de l'État, avait été mobilisée, sans succès, contre le communisme dans les années 1920 après l'avoir été, plus positivement, contre le «complot» du général Boulanger ou de Déroulède. Seules les juridictions ordinaires interviennent, mais recomposées dans leurs compétences. Les cours d'assises sont largement écartées, comme on le voit en général lors des crises politiques et en particulier dans les années 1920 ou à la fin de la $\mathrm{III}^{\mathrm{e}}$ République contre les communistes. Elles apparaissent trop aléatoires, trop incontrôlables, trop sensibles aux mouvements d'opinion et aux pressions des partisans des accusés. Leur marginalisation bénéficie aux tribunaux militaires, parfois qualifiés de juridictions d'exception permanentes ordinaires en raison de leur composition strictement contrôlée et de leur procédure protégeant mieux les secrets d'État, bien que la IV ${ }^{\mathrm{e}}$ République se montre soucieuse d'en atténuer le caractère dérogatoire par la présence de magistrats civils qui les président conformément à la réforme de 1927 et en assument l'instruction pour quelques affaires politiquement sensibles. De plus en plus, depuis la Première Guerre mondiale et les années 1939/1940, les juridictions militaires assument la défense de l'État et élargissent leur compétence au-delà des militaires, en direction des civils et en-dehors des situations de guerre ou d'état de siège. Leur saisie est obligatoire pour les affaires d'atteinte à la sûreté extérieure de l'État et facultative pour les atteintes à la sûreté intérieure de l’État. Pour la période qui nous intéresse, ils interviennent dans les affaires de militaires et d'espions, mais aussi de civils dont les gouvernements veulent dramatiser le sort, soit les auteurs de sabotage de matériels de guerre et, en 1952-1953, quelques dirigeants communistes.

La justice civile professionnelle de droit commun (tribunaux correctionnels et cours d'appel) reste la plus mobilisée, car elle évite les cours d'assises avec leur imprévisibilité et économise le recours aux tribunaux militaires avec leur manque traditionnel de moyens et leur charge politicosymbolique lourde dans une République qui s'en défie. Elle autorise la prise en charge d'une masse d'affaires de plus ou moins faible importance que les gouvernements rattachent à un " plan concerté » de subversion, au sens large puisqu'il englobe les atteintes à la sûreté de l'État, le sabotage, la démoralisation de l'armée, mais aussi la diffamation, la diffusion de 
fausses nouvelles, les campagnes de tracts, les arrêts de travail ${ }^{3}$. Elle offre, enfin, une procédure plus discrète pour traiter les accusations de complot imprudemment engagées contre l'extrême droite avec le "Plan Bleu »" destiné initialement à un jury populaire ou contre les responsables communistes renvoyés devant les militaires.

Enfin, la période est gérée largement, mais non totalement, dans le cadre de la législation existante. Même si André Marie, alors ministre de la Justice, estime que le cadre des lois en vigueur convient mal à la répression des agissements de complot dénoncés par Jules Moch, et demande à l'Assemblée nationale d' " armer la République de textes dont le champ sera moins limité et l'application moins délicate " ${ }^{5}$, le gouvernement ne propose que quelques projets de textes sans rétroactivité condamnable au regard des principes juridiques. Projets que l'Assemblée nationale, encore plus hésitante, adopte partiellement. Le plus radical, le plus politique visant à réprimer tous les actes de violence et voies de fait contre le jeu normal des organismes constitutionnels ne passe pas. Seules sont promulguées deux nouvelles lois. Bien que les communistes les qualifient de «scélérates » et engagent contre elles une véritable guerre physique à l'Assemblée nationale, elles ont des effets limités et relèvent de l'affirmation classique de l'État plutôt que de la lutte contre une subversion au service de l'étranger. La première, temporaire, mais d'application immédiate, de décembre 1947, accélère et alourdit la répression pénale des actes de destruction et de violence en temps de grève ; la deuxième, de mars 1950, relative au sabotage, vise à «donner à la justice un instrument d'action plus maniable et plus léger » que les dispositions du Code pénal trop restrictives en matière de sabotage par omission et de complicité et prévoyant une peine trop disproportionnée pour être applicable, la peine de mort. La Guerre froide est appréhendée, au pire, à partir du capital accumulé des lois d'exception promulguées depuis le $\mathrm{XIX}^{\mathrm{e}}$ siècle en évitant celles initiées par Vichy (notamment, celles promulguées lors des événements de 1848, de la lutte contre les ligues d'extrême droite en 1935-1936 ou contre les communistes et les espions à la fin de la III ${ }^{\mathrm{e}}$ République). Elle est également l'occasion d'un large recours aux procédures judiciaires ordinaires les plus dérogatoires, tels le flagrant délit et la détention provisoire.

3. Circulaire du Ministre de l’Intérieur aux préfets du 17 février 1950.

4. Augustin Jean-Marie, Le plan Bleu-1947, un complot et la République, La Crèche, Geste éditions/histoire, 2006.

5. Le Monde, 20 novembre 1948. 


\section{UN DISPOSITIF ÉTATIQUE ORDINAIRE D’EXCEPTION}

Pour faire face à la situation, le fonctionnement de la justice civile n’est pas bouleversé, mais connaît, là encore, des aménagements classiques en temps de crise. Est ainsi renforcé le pouvoir du Garde des Sceaux et assisté des directions de la Chancellerie sur le Parquet, dont la dépendance hiérarchique, installée par Napoléon I ${ }^{\mathrm{er}}$, n’a pas été remise en cause dans la Constitution de 1946 et échappe aux velléités d'autonomisation de la fin des années 1940 dès qu'il s'agit de "crimes et délits de nature, par leur caractère et leur gravité, à troubler l'ordre public, ou à poser un problème politique sérieux » (Circulaire du 31 mars 1949). Les parquets sont soumis à une obligation classique d'informer, mais considérablement renforcée comme le montrent diverses circulaires ministérielles précisant que « tous les crimes et délits intéressant la sûreté de l'État doivent être immédiatement signalés à ma Chancellerie, au besoin par télégramme ou téléphone ». Elles imposent, lors des phases les plus conflictuelles, l'envoi, hebdomadaire puis mensuel, de l'état des procédures en cours pour « toutes les affaires intéressant la sûreté de l'État ou le maintien de l'ordre public ». État succinct qui « ne saurait exclure des comptes-rendus spéciaux par rapports séparés pour toute affaire importante ". Plus qu'à un devoir d'informer, c'est à un véritable pouvoir de contrôle et de direction que le Parquet est soumis par voie de circulaires collectives et d'instructions particulières qui, comme lors de chaque crise, se multiplient, sont en partie secrètes et vont au-delà de ce que la loi autorise. Elles lui imposent de reconnaître à la Chancellerie et, on le verra, à l'administration des prérogatives exorbitantes. Elles lui font perdre tout pouvoir d'apprécier l'opportunité des poursuites et des appels, seul lui reste celui de donner des avis et d'assurer qu'il est prêt à appliquer les instructions ministérielles, même contraires.

Non seulement, elles ne cessent d'appeler à la plus grande sévérité et à la plus grande célérité, mais elles prescrivent de poursuivre ou de ne pas poursuivre, imposent des textes et des jurisprudences à viser, les appels à interjeter, les peines à requérir. Le ministre sanctionne parfois, comme on le voit pour le procureur de Béthune, déplacé et rétrogradé, pour ne pas avoir appliqué le flagrant délit prescrit ; il réprimande souvent et vertement : « Je n'accepte absolument pas la décision rendue emprunte d'une inadmissible faiblesse. Faire appel a minima. Dans mes instructions noter que je m'étonne que le Parquet devant la gravité des faits, ait songé - et le procureur général ensuite - à accepter une décision aussi indéfendable par sa faiblesse ${ }^{6}$. Les affaires d'atteinte à la sûreté de l'État les plus graves

6. Lettre aux procureurs généraux Amiens AN, BB/18/3731. 
sont encore plus contrôlées : elles sont centralisées en général à Paris, exceptionnellement aussi à Toulon en 1952-1953, et prises en charge par des magistrats spécialisés proches du politique, tel le juge d'instruction de l'affaire du «Plan Bleu » très lié aux ministres socialistes Jules Moch et Edouard Dupreux, ou très disciplinés à l'image du procureur supervisant l'affaire du "complot» de Toulon, qui transmet à la Chancellerie des comptes-rendus quotidiens avec envoi systématique de tous les documents intéressant l'information, même ceux couverts par le secret de l'instruction. La tendance à la centralisation des affaires de "complot », déjà repérable lors de la répression des communistes dans les années 1920, produit des effets importants sur la justice "d'en bas » : elle cantonne ses servants dans l'approvisionnement de leurs collègues parisiens et dans les incriminations pénales plus ordinaires. Ainsi, les instructions télégraphiées du ministre de la Justice aux procureurs généraux de province à la suite des manifestations contre le général américain Ridgway précisent qu’ils ne doivent pas ouvrir d'informations pour atteinte à la sûreté intérieure de l'État réservées au Parquet de Paris : il leur revient de « veiller (à l') application particulièrement stricte et ferme " d'informations ouvertes pour infraction à la loi du 7 juin 1848 sur les attroupements et toutes les infractions connexes (notamment celles prévues par la loi du 10 janvier 1936 pour port d'armes prohibées et par le décret du 25 octobre 1935 portant réglementation des mesures relatives au renforcement du maintien de l'ordre public), de maintenir les délinquants arrêtés en détention préventive et de recueillir les éléments susceptibles de renforcer les charges ayant motivé les instructions données au parquet de la Seine. Enfin, dernier aménagement, l'étatisation de la justice est renforcée. Comme à chaque crise, prédominent le ministre de l'Intérieur et les préfets, auxquels la IV République ajoute une innovation mineure, les IGAME. Sans doute n'ontils pas autant de prérogatives que dans d'autres circonstances. S'ils conservent leurs attributions exorbitantes, excluant largement la justice, en matière d'expulsion des étrangers et de traitement de l'espionnage, ils ne détiennent plus le pouvoir d'internement administratif qui évite ou annule l'intervention des juges et que la III $^{\mathrm{e}}$ République finissante, Vichy, la Libération et la guerre d'Algérie leur a reconnu ou leur reconnaîtra. Ce qui rend la justice avec son pouvoir de sanction d'autant plus stratégique. Et d'autant plus importante à contrôler.

La dépendance de la justice envers les autorités administratives et policières, déjà perceptible en temps ordinaires, est considérablement accrue. Elle en est tributaire pour la connaissance des affaires, comme en témoignent les plaintes répétées des procureurs généraux reprochant à la police de les informer trop tard pour appliquer la procédure privilégiée de flagrant délit. Elle en dépend encore pour la protection des magistrats et des au- 
diences menacés, ou encore pour l'exécution des décisions de justice contestées. Les circulaires ministérielles, quant à elles, ne cessent de prescrire aux chefs des parquets locaux de nouer un « étroit contact » avec les préfets, de les tenir régulièrement informés, d'assister aux réunions qu'ils organisent, d'« appuyer leur action par toutes les voies légales ». Elles prévoient même de leur reconnaître certaines prérogatives exorbitantes accordées de fait et par tradition au ministre de la Justice, comme l'accord préalable pour certaines réquisitions ou la levée du secret de l'instruction. Elles invitent à trouver dans les documents des Renseignements généraux qu'elles fournissent ou invitent à aller chercher les informations nécessaires aux décisions judiciaires (circulaires du 30 décembre 1948 et du 21 février 1950).

Significatif du pouvoir de direction politique et de pré-construction juridique du ministère de l'Intérieur, la circulaire confidentielle du ministre de la Justice transmettant aux procureurs généraux une circulaire, tout aussi secrète, du 17 février 1950, où le ministre de l'Intérieur parle aux préfets ${ }^{7}$ d' « un véritable plan concerté » menaçant l'État, leur prescrit de se mettre en rapport avec les autorités militaires locales et les procureurs généraux pour faire poursuivre systématiquement tout ce qui s'y rapporte (diffamations, injures, fausses nouvelles...) et leur donne, en annexe, toutes les qualifications juridiques à retenir (sabotage, pillage...) avec référence des lois et articles du Code pénal. Comme lors de chaque crise, les préfets reconduisent des pratiques témoignant de leur propension à diriger les magistrats comme des fonctionnaires : ils les font surveiller par les Renseignements généraux, les convoquent pour discuter des affaires, écrivent au ministre de l'Intérieur pour leur reprocher de manquer de sévérité, de mal les «seconder » et pour réclamer des sanctions. Tel le préfet de police de Paris écrivant, en 1953, au ministre de l'Intérieur qui transmet à son collègue de la justice son rapport : «... je crois que de très sévères observations doivent être adressées aux trois Magistrats ayant rendu l'arrêt... et je vous serais très reconnaissant de vouloir bien envisager la possibilité d'intervenir dans ce sens auprès de M. le Garde des sceaux - Ministre de la Justice $»^{8}$.

La justice bénéficie toutefois de garanties spécifiques que tous les régimes, même celui de Vichy, reconnaissent et ménagent sous peine d'être accusés d'arbitraire. Au principe de l'indépendance de la justice, la IV République y tient par ses valeurs et y est tenue par la concurrence entre les démocraties occidentales et les démocraties populaires de l'Est. Elle est à l'origine d'une innovation qui conforte de manière inédite la marge d'autonomie des magistrats qui jugent. Si le Parquet demeure toujours sous la dépendance du ministre, le Siège échappe, pour la première fois, à sa tu-

7. Pour les deux circulaires, voir AN, BB/18/3995.

8. AN, BB/18/4167. 
telle et relève d'un nouveau Conseil supérieur de la magistrature dont le Garde des Sceaux n'est que le vice-président, la présidence revenant au président de la République et les autres membres étant des magistrats élus par leurs pairs et des personnalités désignées par l'Assemblée et le président de la République. Cette nouvelle organisation a des effets directs sur la gestion des affaires relevant de la Guerre froide. Alors qu'au cours d'un conseil des ministres d'avril 1950, plusieurs membres du gouvernement s'étonnent vivement de plusieurs jugements favorables aux communistes, Vincent Auriol, très attaché au CSM, fait remarquer que la question ne relève plus du conseil des ministres, mais du CSM, seul chargé d'assurer la discipline du siège et la garantie de son indépendance : les ministres demandent alors de «faire savoir au CSM que la justice entrave notre action politique ». Lequel répond que les décisions des magistrats du Siège relèvent de leur seule conscience et, le cas échéant, d'une cour d'appel et qu'il n'appartient à personne d'en censurer les motifs ${ }^{9}$. Sa protection ne doit pas être toutefois surévaluée. Outre sa composition à majorité politique, ses moyens face à l'alliance du ministre de la Justice et des magistrats des directions du ministère et du Parquet sont très limités et sa conception de l'indépendance reste restrictive, en témoigne sa réaction après l'attentat contre le président de la chambre des mises en accusation ayant remis en liberté Jacques Duclos.

\section{LE TRAVAIL DE BANALISATION DES JUGES}

Il est difficile d'évaluer exactement l'activité de la magistrature civile en raison du flou des affaires concernées, de la multiplicité des incriminations juridiques mobilisées : en témoignent la variété des affaires relevant du «plan concerté » de subversion, évoqué dans la circulaire du ministre de l'Intérieur destinée aux préfets et diffusée auprès des procureurs généraux en février 1950 et les difficultés des parquets à remplir pour la Chancellerie les états des affaires dites de «maintien de l'ordre et d'atteinte à la sûreté de l'État»; certains mentionnent des affaires d'abattage d'arbres et de manifestations à la suite d'expulsion de métayers. Il existe de surcroît des divergences, des oppositions, entre magistrats du Parquet et du siège, exacerbées par la réforme du CSM qui autonomise les juges. Ou encore, entre tribunaux et parfois au sein d'un même tribunal. Beaucoup de débats jurisprudentiels, d'autant plus essentiels qu'il s'agit surtout d'adapter des textes anciens à une situation nouvelle, ne sont pas clos par la Cour de cassation à qui il revient d'unifier la jurisprudence.

9. Le Monde des 23-24 et 28 avril 1950. 
Si de nombreuses décisions de justice suscitent des dénonciations enflammées de la part des communistes, d'autres, moins nombreuses, mais suffisamment fréquentes pour être significatives, donnent lieu à "l'étonnement réprobateur » de ministres se plaignant que la justice entrave leur action, à des rapports réprobateurs de préfets et du préfet de police de Paris, transmis et appuyés par le ministre de l'Intérieur, où il est souligné « une fois de plus, s’il en était besoin, la carence de la plupart des magistrats et de leur crainte des responsabilités » ${ }^{10}$; « À plusieurs reprises déjà, j'ai été appelé à attirer votre attention sur l'état d'esprit, que l'on peut qualifier de fâcheux, dont certains magistrats paraissent faire preuve dans différentes affaires présentant incontestablement un aspect politique ${ }^{11}$. En 1948, Jules Moch rapporte à son collègue de la justice qu'un préfet se demande si, à l'avenir, il ne conviendrait pas d'éviter les plaintes des préfets qui se retournent contre eux puisque le « résultat final tant à démontrer aux émeutiers qu'ils n'ont rien ou pas grand-chose à redouter... En me faisant l'écho des doléances du Préfet, je ne fais que traduire un malaise qui tend à se répandre parmi les fonctionnaires d'autorité et que j'ai le devoir de dissiper. Mais il est bien évident que je ne peux arriver à ce résultat qu'avec votre concours. Je me permets donc d'insister pour que vous recommandiez à nouveau aux procureurs généraux de faire preuve d'une vigilance particulière dans la répression des actes constituant des atteintes graves au bon ordre public ${ }^{12}$.

Les parquets font très souvent appel contre des jugements de première instance coupables de « faiblesse ". Sans être toujours suivis par les cours d'appel qui confirment souvent les décisions contestées, quelquefois les adoucissent. Au niveau de leur ressort, les procureurs généraux doivent engager tout un travail visant à briser la persistance de jurisprudences installées ou à empêcher la constitution de nouvelles jurisprudences contraires aux directives ministérielles. Les tensions, les conflits entre les juges et le gouvernement seraient beaucoup plus fréquents si les procureurs, tout en reconnaissant la trop grande indulgence de certaines décisions, conseillaient de ne pas faire appel afin d'éviter des désaveux solennels aux conséquences plus graves. Ils font valoir à la Chancellerie qu'il vaut mieux un classement de leur part qu'une relaxe d'un tribunal, dotée de la solennité et de la publicité d'un jugement, qu'il est préférable de ne pas requérir l'annulation d'un jugement trop clément, mais discret d'un petit tribunal qu'en appeler à une cour d'appel, plus élevée dans la hiérarchie judiciaire et juridique et dont les arrêts sont plus susceptibles de faire jurisprudence,

10. AN, BB/30/1772.

11. Rapport du préfet du Vaucluse de 1950. AN, BB/18/4167.

12. Rapport du préfet de police de Paris de 1953. AN, BB/18/3732. 
disposée à confirmer. Le ministre de la Justice, pour sa part, ne cesse de devoir multiplier les circulaires, rappelant la nécessité d’une sévérité exemplaire, car son « attention est appelée sur la faiblesse en matière de... dont ont fait preuve diverses décisions récentes » (circulaire du 14 septembre 1949); prescrivant d'être moins sensibles aux pressions locales (circulaire du 4 octobre 1949), de mieux assurer la police des audiences afin de plus protéger les policiers qui déposent lors des procès (circulaire du 11 septembre 1952), de moins s'adresser aux maires et plus aux juges de paix et aux commissaires de police pour obtenir les renseignements de moralité (circulaire du 5 septembre 1949).

Domine en fait la tendance à ramener les affaires relevant ou pouvant relever de la Guerre froide à des questions ordinaires d'ordre public national envers lesquelles ils montrent une certaine sévérité ou à des problèmes d'ordre politique et syndical envers lesquels les magistrats manifestent, depuis le $\mathrm{XIX}^{\mathrm{e}}$ siècle, réserve et modération. Tendance particulièrement sensible "en bas ", où les magistrats sont directement soumis aux pressions, aux violences, et confrontés aux circonstances particulières de chaque affaire, où il est encore plus difficile de faire fonctionner la catégorie, déjà non redéfinie, inadaptée, de complot avec des inculpés trop ordinaires, sans histoire, souvent pris au hasard parmi beaucoup d'autres manifestants ou grévistes, ayant agi sous le coup de la colère, entraînés par une foule suivant des mots d'ordre dont les auteurs ne sont pas poursuivis. Tendance, enfin, d'autant plus marquée que l'on sort de la période «chaude », comme le montre l'affaire dite du «complot des pigeons » visant Jacques Duclos et quelques autres responsables communistes: commencée en 1952 devant le tribunal correctionnel de Paris au titre de l'atteinte à la sûreté intérieure de l'État, continuée en 1953 devant le tribunal militaire au nom de l'atteinte à la sûreté extérieure de l'État, elle se termine trois ans et demi plus tard devant une chambre correctionnelle ordinaire condamnant à des peines avec sursis des inculpés poursuivis pour des délits plus classiques (rébellion et insultes à agents aggravés, pour certains, de port d'armes et de coups et blessures).

Les juges ne sont pas disposés à consacrer d'eux-mêmes les dérogations exceptionnelles que le gouvernement attend, mais que le parlement hésite ou refuse d'officialiser, comme on le voit pour l'application du flagrant délit aux députés communistes. Dans les deux affaires qui font, en la matière, scandale, le tribunal de Brest et la Chambre des mises en accusation de Paris ne font pas œuvre de rébellion et de parti pris comme ils en sont accusés : ils ne se prononcent pas sur le fond, c'est-à-dire sur la responsabilité des inculpés dans les manifestations et les violences, ils refusent d'assumer ce que l'Assemblée nationale n’a jamais décidé, restreindre l'immunité des députés communistes. Il existe sans doute quelques juris- 
prudences innovantes, extensives qui adaptent les textes anciens aux nouvelles accusations et que la chancellerie diffuse par circulaires, mais elles restent le plus souvent débattues et demeurent dans le cadre classique de la défense de l’État et de la liberté du travail.

De manière générale, les magistrats manifestent des réticences à employer les incriminations pénales les plus dérogatoires et les plus politiques. Telle la détention d'armes et de munitions envers laquelle divers tribunaux manifestent de la "faiblesse », alors qu'il est, selon le ministre de la Justice, « évidemment impossible d'assurer qu'aucun usage répréhensible ne sera jamais fait de ces armes et munitions, même s'il est allégué qu'elles ont été conservées par négligence et sans intention criminelle » (circulaire du 14 septembre 1949). Tel encore le sabotage, que le gouvernement réforme pour favoriser son usage par des juges jusqu'ici hésitant à élargir sa définition restrictive, jugeant disproportionnée la peine de mort prévue et préférant, en matière sociale, le délit d'entrave à la liberté du travail réprimant l'opposition à l'activité d'ouvriers ne voulant pas faire grève. Même après la nouvelle loi, le tribunal militaire qui juge Henri Martin, jeune militaire communiste, abandonne l'accusation de sabotage au bénéfice de la "minorité de faveur » (3 juges sur 7) et le condamne, seulement à la majorité, pour démoralisation de l'armée. Même cette dernière inculpation, souvent initiée par le ministre de la Défense, est l'objet de la part de tribunaux civils d'interprétations restrictives. En considérant, par exemple que les propos antimilitaristes visaient seulement les soldats et conscrits ayant choisi de faire la guerre en Indochine, et qu' « il n'y est pas question d'inviter des militaires quels qu'ils soient à désobéir à leurs chefs », et « que par voie de conséquence le fait d'essayer de détourner des militaires d'un engagement nécessaire au recrutement des troupes coloniales ne porte nullement atteinte à la discipline que doit observer tout militaire dans l'exercice de son service normal, et partant ne peut être considéré comme entreprise de démoralisation. Que, dès lors, tous les éléments des délits reprochés aux inculpés n'étant pas réunis, ceux-ci sont en voie de relaxe $^{13}$; ou de déqualifications juridiques, comme le fait le tribunal de Nantes qui condamne à 30 jours d'emprisonnement avec sursis pour abandon de poste un conducteur de train, secrétaire local du syndicat des conducteurs obéissant à un mot d'ordre de la CGT cheminots, ayant arrêté 45 secondes son train parce que deux wagons servaient à transporter du matériel de guerre ${ }^{14}$.

Certaines cours d'appel, il est vrai, censurent de telles interprétations restrictives. Telle la cour de Dijon annulant, en décembre 1950, une relaxe

13. Tribunal correctionnel de Montpellier, audience du 28 mars 1950.

14. Le Monde, 2 juin 1950. 
prononcée deux mois auparavant par le tribunal correctionnel de la même ville, pour délit non nettement caractérisé, en faveur du secrétaire de l'Union des jeunesses républicaines de France ayant envoyé aux conscrits une lettre avec bulletin d'adhésion et une chanson ronéotée dont le refrain était : "Rien à faire pour la sale guerre. Rien à faire nous ne marcherons pas ». La cour condamne l'inculpé à 3 mois plus 20000 francs d'amende, en reprenant les réquisitions du Parquet rappelant que la jurisprudence assimile les conscrits à de jeunes soldats et que la chanson équivaut à une incitation de militaires à la désobéissance.

Quand ils condamnent la destruction de numéros du Figaro, les tribunaux le font comme si c'était un simple vol, pour soustraction frauduleuse et dommages à la propriété d'autrui. Quand ils jugent un conflit privé comme celui opposant David Rousset et Les Lettres françaises, ils le traitent comme un conflit idéologique classique pour lequel ils ont une tradition de réserve : le tribunal de la Seine (12 janvier 1951) et la cour d'appel de Paris (6 juillet 1953) tiennent compte, pour apprécier la gravité de la diffamation et l'importance du préjudice, du « caractère largement idéologique du différend », « de la personnalité du plaignant et de ses adversaires ». Le tribunal de la Seine retient même le « caractère largement idéologique » de la publication du jugement. Ce qui les conduit à une « application modérée de la loi pénale et une évaluation mesurée des dommages-intérêts » ${ }^{15}$.

Comme ils ont l'habitude de le faire, les tribunaux condamnent plus facilement et plus sévèrement les actes de violence contre les personnes et surtout les forces de l'ordre. Et encore, ne témoignent-ils pas à chaque fois d'une grande énergie répressive. Plutôt qu'en défenseurs du moral des armées et remparts contre la subversion, ils se posent en gardiens solennels, à l'occasion sentencieux, de la souveraineté de la loi, de la protection des agents de l'autorité de l'État et des limites à l'usage des libertés fondamentales, comme la liberté du travail ou d'opinion. La cour d'appel de Nancy, confirmant la condamnation d'un jeune manifestant ayant bousculé un gendarme conduisant au palais de justice un inculpé qui en profite pour fuir, précise ainsi dans ses attendus: "Il ne peut être toléré qu'un citoyen s'arroge le droit d'intervenir dans l'exercice des fonctions des agents de l'autorité chargés d'exécuter les mesures de justice, et il ne lui appartient pas d'en apprécier la légalité selon ses opinions personnelles. Une pareille immixtion ne peut trouver sa justification en des théories politiques... Si la liberté d'opinion est illimitée, la liberté d'action est subordonnée au respect de la loi » ${ }^{16}$.

Les peines, elles-mêmes, ne sont pas à la hauteur d'une accusation de complot, et ne correspondent pas à une répression exemplaire d'État exclu-

15. Le Monde 7 juillet 1953.

16. Le Monde, 10-11 septembre 1950. 
sivement soucieuse de la gravité des faits et de l'intérêt général de la répression. Non seulement, il y a des relaxes, peu nombreuses, mais politiquement lourdes, vécues comme un désaveu de la politique du gouvernement et une victoire communiste, mais les condamnations les plus fréquentes consistent en des amendes et surtout des peines d'emprisonnement modérées (de quelques jours à un, deux ou trois mois, rarement plus), souvent assorties du sursis pondéré d'une amende, soit le type de condamnations que le ministre de la Justice dénonce comme manquant de l'effet dissuasif attendu et contre lesquelles il prescrit au parquet de faire appel.

Symptomatique d'un travail de banalisation et de modération, les juges font massivement jouer les circonstances atténuantes attachées à la personnalité des accusés et à la singularité des situations. Elles sont au cœur du pouvoir de juges soumis à la souveraineté de la loi et spécialisés dans l'application concrète de lois générales et abstraites, au cœur de l'ambivalence d'un habitus professionnel partagé entre la rigueur impersonnelle de la défense de la loi, de l'ordre, et l'appréciation singulière et humaine des situations. Les circonstances atténuantes reconnues sont traditionnelles (absence d'antécédents judiciaires, bonne réputation, situation familiale, bonne tenue et regrets sincères à l'audience...), complétées d'une dimension nouvelle mettant à mal l'image du communiste au service de l'étranger : le passé de résistant que les juges n'hésitent pas à faire jouer pour les militants communistes déportés ou décorés et dont ils poussent parfois très loin les effets. Il peut justifier l'octroi du sursis comme le fait le tribunal de Valenciennes en faveur de deux conseillers municipaux communistes poursuivis à la suite d'une plainte du ministère des Armées pour avoir déclaré que les combattants d'Indochine «méritaient 12 balles dans la peau ", paroles pourtant très graves selon ce même tribunal puisqu'il les qualifie d' " atteinte intolérable à l'esprit de la patrie » ${ }^{17}$. Certains juges en font une véritable excuse absolutoire, mieux un mobile légitime. Tel le tribunal de Grasse acquittant deux anciens déportés poursuivis pour avoir détruit des numéros du Figaro au prétexte que « les deux prévenus, ayant eu à souffrir de la barbarie nazie, avaient obéi à un réflexe irrésistible en ce jour de la libération des camps de la mort ${ }^{18}$. Tel encore ce juge unique parisien relaxant le rédacteur en chef de L'Humanité, arrêté et poursuivi pour vol après avoir brûlé des numéros du Figaro, au motif que « la destruction sur la voie publique d'une publication éditant un article subversif de nature à troubler l'ordre public, ou à constituer une provocation au sacrifice de ceux qui ont pris part à l'activité douloureuse de la libération de

17. Le Monde, 10 octobre 1951.

18. Le Monde, 17 mai 1950. 
la France, article qui a échappé à la censure vigilante des pouvoirs publics, ne constitue pas, en l'absence d'intention frauduleuse, un délit de vol ${ }^{19}$.

Dernier élément qui confirme que les magistrats se situent dans l'ordre classique du maintien de l'ordre et des affaires politico-syndicales, l'importance jouée, spécialement au niveau local et même des cours d'appel pourtant plus sensibles aux exigences juridiques, par un argument d'opportunité, avoué parfois dans les attendus des jugements et surtout dans les rapports des Procureurs généraux expliquant au ministre les motivations implicites des juges : "l'apaisement», «l'esprit d'apaisement social ». Il explique que les tribunaux de première instance condamnent souvent avec sursis et que les cours d'appel confirment, voire adoucissent, les décisions frappées d'appel par le Parquet. Soit par volonté délibérée, soit par réalisme résigné, parce qu'elles n’ont " pas cru devoir modifier les premières peines dans un but d'apaisement ». Même les procureurs généraux et, plus encore, les procureurs de la République les plus immédiatement confrontés aux situations, font valoir un tel argument auprès de la chancellerie, souvent sur le registre de l'impuissance devant l'indulgence prévisible des juges d'instance et d'appel. À moins que ne soit sous la caution des préfets également préoccupés de ne pas ranimer les conflits.

On est ainsi très loin de l'attitude de la magistrature dans les années 1920 où la jurisprudence la plus solennelle, celle de la Cour de cassation, se substitue à un législateur jugé défaillant et assimile le communisme à l'anarchisme défini par les lois «scélérates» de 1893-189420. Soit une infraction pénale non pas politique, dirigée contre une forme de gouvernement et méritant un traitement modéré spécifique, mais relevant de la « protection sociale », portant atteinte aux fondements mêmes de la société et justifiant une sévérité répressive exemplaire de droit commun. On est encore plus loin des magistrats des juridictions d'exception de Vichy qui appliquent une législation dérogatoire confondant dans un même sort communisme et anarchisme assimilés au "terrorisme ", à une "subversion » à la fois sociale et nationale, et qui condamnent à des peines d'une sévérité extrême des communistes pour des faits d'une gravité incertaine. Tels les membres de la Section spéciale de Paris prononçant, lors de leur première audience en 1941, la peine capitale contre trois communistes auteurs de simple propagande ou ceux du Tribunal spécial de Toulouse condamnant à mort, en 1943, un résistant communiste, d'origine juive polonaise et ancien des Brigades internationales, accusé de complot international et d'attentat pour avoir réceptionné une valise d'explosifs : « Si

19. Le Monde, 20 avril 1950.

20. Monier Frédéric, « Des menées anarchistes aux menées communistes : magistrats et ordre social », Cahiers Jean Jaurès, juillet-septembre 1996, nº 141, p. 49 sq. 
l'on considère, écrit le Procureur général appelant à l'exécution de la sentence, que cette valise a suivi jusqu'à lui une carrière qui suppose le concert et l'organisation entre juifs en état d'insurrection contre l'ordre établi on ne peut qu'assimiler le cas du condamné à celui d'un individu qui aurait finalement réalisé son dessein d'attentat ». Une telle attitude, il est vrai, ne perdure pas jusqu'à la fin de Vichy : les magistrats veulent de moins en moins assurer une répression leur valant des menaces, pire des exécutions, et admettent de plus en plus le caractère patriotique et désintéressé de l'action des communistes, du moins quand ils ne commettent pas d'attentats contre des autorités françaises.

\section{UNE CRITIQUE RÉCURRENTE : LE MANQUE DE SENS DE L'ÉTAT DES MAGISTRATS}

La période étudiée révèle les effets d'un habitus professionnel qui ramène l'extraordinaire politique, ici le communisme et la Guerre froide, à l'ordinaire pénal. Soit à une question classique d'ordre public pour laquelle le magistrat intervient, comme le disait déjà un haut magistrat sous Vichy, « en tenant compte de la défense nécessaire d'une société qui a le droit et le devoir de se prémunir contre tous les éléments de désordre, mais qui fidèle à la tradition française, ne perd jamais de vue les circonstances atténuantes ${ }^{21}$. Soit à une affaire d'ordre politique ou syndicale, objet d'un traitement spécifique de moindre rigueur. Cette dernière appréciation, marquant une évolution par rapport aux années 1920, a été renforcée par les aléas historiques du statut réservé aux communistes : poursuivis dans les années 1920, quasi intégrés à l'exercice gouvernemental avec le Front Populaire, poursuivis de nouveau à la fin de la $\mathrm{III}^{\mathrm{e}}$ République et sous Vichy, participant au gouvernement à la Libération où ils profitent de l'annulation des condamnations prononcées sous Vichy, occupent, au début, la responsabilité du ministère de la Justice, participent aux commissions de réforme de la chancellerie et à la commission d'épuration de la magistrature retenant comme grief principal de sanction la répression contre les résistants, en particulier communistes.

L'évolution du statut judiciaire des communistes est facilitée par les liens personnels qui se sont établis entre des communistes et des magistrats. On n'a plus affaire à deux mondes qui s'ignorent. Il ne s'agit pas

21. Discours du premier président de Pau lors de l'installation du nouveau procureur général le 10 novembre 1943. Cour d'appel de Pau, audience solennelle, Imprimerie commerciale, Pau, 1943. 
d'entrisme comme on le voit ou le soupçonne pour d'autres corps d'État. Les nominations stratégiques réalisées par l'avocat communiste Marcel Willard lors de son passage à la tête de la chancellerie sont rares et assez vite neutralisées. Ce qui s'est noué et qui demeure, ce sont les liens entre des juristes communistes et certains nouveaux hauts magistrats parisiens pendant la Résistance (en particulier, au sein du Front national des juristes initié par des avocats communistes ${ }^{22}$ ) et à la Libération par le truchement de la chancellerie. Sous la IV République, cette solidarité se poursuit, bien que malmenée par la Guerre froide, au sein d'organisations (le Mouvement national judiciaire, l'Association des magistrats résistants, l'Association des juristes démocrates). Dans ses Mémoires ${ }^{23}$, l'avocat communiste Joë Nordmann raconte qu'à sa réception célébrant ses vingt ans de parti, en 1953, étaient présents des hauts magistrats qu'il avait connus lors de ses fonctions de directeur de cabinet de Marcel Willard. Il mentionne également son amitié personnelle avec le président du tribunal de Périgueux, l'existence d'un magistrat qui n'ose rien lui refuser et, surtout, la signature par certains des plus hauts magistrats de l'appel pour la paix de Stockholm : le procureur général et trois présidents de chambre de la Cour de cassation. Des rapports de police rapportent les liens de magistrats « d'en bas » avec les organisations satellites du PCF, tel le président du tribunal de Niort: président des combattants pour la paix, président d'honneur de l'ARAC, président d'honneur du secours populaire français, il aurait conseillé des prévenus poursuivis à la suite d'une manifestation ${ }^{24}$. D’autres dénoncent le ton complaisant de magistrats envers les inculpés communistes, à l'image du président du tribunal d'Orange jugeant, en février 1950, quatre cheminots ayant arrêté un train lors d'une grève de la CGT : "C'est avec une singulière bonhomie, mettant dans l'aisance et les inculpés et les témoins communistes à décharge, que M. B., Président du Tribunal d'Orange, a dirigé les débats... (aux inculpés, il aurait indiqué) sur un ton familier les faits reprochés. À plusieurs reprises par la suite, il laisse entendre que ces faits ne constituent pas une entrave à la liberté du travail, mais seulement un acte contraventionnel. (Il aurait ajouté) « Vous n'avez pas à avoir peur de la sentence de ce tribunal, vous me connaissez, pendant l'occupation, malgré certaines pressions, je n'ai jamais poursuivi les résistants, les juifs ou les communistes. Aujourd'hui pas plus qu'hier, je ne me laisserai influencer par personne pour rendre la véritable justice »... Quant aux témoins de moralité, tous communistes, ils sont venus à la barre

22. Israël Llora, Robes rouges années noires, Paris, Fayard, 2005.

23. Nordmann Joë, Anne Brunel, Aux vents de l'histoire, Arles, Actes Sud, 1996, p. 339.

24. AN, BB/30/1772. 
surtout pour développer les thèmes habituels du PCF mais à deux reprises, cependant, le Président B. les a interrompus avec courtoisie ${ }^{25}$.

Les critiques qui dominent contre les magistrats ne renvoient cependant pas à une trop grande proximité politique avec les communistes, plutôt au manque de sens de l'autorité et de la sévérité d'État, au défaut de courage d'État. C'est ce que leur reprochent les préfets, on vient de le voir. C’est encore ce que dénoncent les hommes politiques, tel René Mayer, alors ministre de la Justice, leur rappelant qu'ils doivent savoir résister aux pressions, rester insensibles « aux bruits de la rue ${ }^{26}$, ou encore MartinaudDuplat, alors avocat président administratif du parti radical et futur ministre de la Justice au moment de l'affaire Duclos : «Un gouvernement digne de ce nom ne saurait laisser bafouer l'autorité de l'État par une justice qui, parfois, laisse plus apparaître dans ses jugements la peur de l'avenir que la connaissance du droit. Une feinte ignorance paraît à certains tribunaux plus garante de leur tranquillité que le courage civique dans l'accomplissement de leur devoir. Dans le respect de la séparation des pouvoirs, c’est la fermeté des hommes publics qui rendra aux magistrats la conscience de leur mis$\operatorname{sion}^{27}$. » C'est enfin de cette même absence de courage d'État que certains procureurs généraux accusent, auprès du ministre de la Justice, quelques procureurs (il y a « tout lieu de penser que mon substitut - le procureur de Riom - n’avait pas exécuté les instructions que je lui avais données, parce que, incertain de l'évolution de la situation, il craignait, en s'y conformant, d'être éventuellement tenu pour responsable de leur exécution... Pour résumer ma pensée, j'estime que mon Substitut de Riom a agi ainsi par manque de courage ${ }^{28}$ ) et, surtout, les juges du Siège (le même procureur général se plaint de sa cour d'appel « semblant ne pas comprendre la gravité relative des faits et peut-être aussi, soucieuse avant tout, de ne pas s'exposer à des représailles. Cette juridiction a fait preuve d'une indulgence, qui ne pourra qu'être d'un effet déplorable pour les poursuites à venir » ${ }^{29}$.

On retrouve, finalement là, une critique récurrente contre l'incapacité des magistrats à se hisser au niveau de la défense de l'État lors des situations de crise politique, sociale, nationale grave. La confidence acerbe de De Gaulle à propos de sa double expérience décevante de la Libération et de la guerre d'Algérie, en témoigne : « certes, il y a des magistrats courageux avec lesquels on peut faire quelque chose, mais les autres ne veulent pas prendre de risques ; en France, la caste des juges a toujours été mora-

25. AN, BB/30/1772.

26. Le Monde, 20 juin 1950.

27. Le Monde 23-24 avril 1950.

28. AN, BB/18/3733.

29. AN, BB/18/3822. 
lement incapable de juger des menaces contre l’État... En réalité, la Justice est l'expression de la pensée d'une caste sociale, la bourgeoisie qui, dans sa majorité, a perdu le sens de l'État, le sens de la Nation, le sens de la Patrie. Les juges ne cherchent qu'à juger des dossiers civils! Surtout pas une affaire grave, qui menace le pays ! Car ils n'en ont pas le courage ! Ils préfèrent se cantonner dans les litiges de succession ou les braquages ${ }^{30}$. »

30. Peyrefitte Alain, C'était de Gaulle, Paris, Gallimard, 1999, Coll. Quarto, 2002, tome II, p. 131-132. 